\title{
CODED SCENES FOR FAST SYSTEM CALIBRATION IN MAGNETIC PARTICLE IMAGING
}

\author{
Serhat Ilbey ${ }^{\star \dagger} \quad$ Can Barış Top ${ }^{\star} \quad$ Alper Güngör ${ }^{\star} \quad$ Emine Ulku Saritas ${ }^{\dagger \S} \quad$ H. Emre Güven ${ }^{\star}$ \\ * ASELSAN Research Center, 06370 Ankara, Turkey \\ ${ }^{\dagger}$ Department of Electrical and Electronics Engineering, Bilkent University, Ankara, Turkey \\ $\S$ National Magnetic Resonance Research Center (UMRAM), Bilkent University, Ankara, Turkey
}

\begin{abstract}
Magnetic nanoparticle (MNP) agents have a wide range of clinical application areas for both imaging and therapy. MNP distribution inside the body can be imaged using Magnetic Particle Imaging (MPI). For MPI image reconstruction with the system function matrix (SFM) approach, a calibration scan is necessary, in which a single MNP sample is placed and scanned inside the full field of view (FOV), which is a very time consuming task. In this study, we propose the use of coded scenes that include MNP samples at multiple positions inside the FOV, and reconstruct the SFM using compressed sensing techniques. We used simulations to analyze the effect of number of coded scenes on the image quality, and compare the results with standard sparse reconstruction using single MNP sample scan. The results show that with the proposed method, the required number of measurements is decreased substantially, enabling a fast system calibration procedure.
\end{abstract}

Index Terms - Magnetic particle imaging, compressive sensing, system matrix, image reconstruction, alternating direction method of multipliers, sparse reconstruction, magnetic nanoparticles

\section{INTRODUCTION}

Magnetic nanoparticle (MNP) tracers have many potential clinical applications such as angiography, stem cell tracking, molecular and cancer imaging, and hyperthermia therapy and imaging [1-4]. Magnetic Particle Imaging (MPI) was introduced for imaging the MNP density inside the body [5]. This imaging modality uses a static magnetic field called the selection field, with a field free region (FFR). The MNPs inside the FFR can be disturbed by an additional time varying magnetic field (drive field), while the particles outside this region are saturated and non-responsive. The FFR is scanned inside a field of view (FOV) and the response of the MNPs to

This work was supported by the Scientific and Technological Research Council of Turkey (Project number 9050103). The work of EUS was supported in part by the European Commission through FP7 Marie Curie Career Integration Grant (PCIG13-GA-2013-618834), by the Turkish Academy of Sciences through TUBA-GEBIP 2015 program, and by the BAGEP Award of the Science Academy. the drive field is captured by receive coils. Resultant images show the density distribution of the MNPs.

Image reconstruction in MPI can be done operating directly on the time domain data [6], or an inverse problem solution can be sought defining the physical problem with a linear system of equations in the frequency domain [7]. For the latter one, a system function matrix (SFM) is required which relates the measured data to voxel positions. This is usually done by measuring the response of a small volume of MNPs at each position on the FOV grid. By this way, all non-idealities of the imaging hardware system transfer function is included in the calibration process. The drawback of this method is the long measurement times of the calibration process, which takes hours for even a small FOV [8]. To mitigate this problem, compressed sensing methods were proposed for SFM measurement and reconstruction. The rows of the SFM, i.e., the spatial sensitivity distribution of the imaging system, can be represented with a much smaller number of elements compared to the grid size of the image in discrete cosine or Chebyshev transform domains [9]. Therefore, the SFM can be reconstructed with smaller number of measurements at randomly selected calibration positions inside the FOV. Acceptable degradation on the MPI images can be achieved using only $10 \%-20 \%$ of the full grid for the calibration [10]. This rate can be further decreased by using the symmetry of the SFM rows [11]. Although these procedures accelerate the calibration process, calibration durations may still be a problem for large FOVs needed in clinical scans.

In this study, we propose the use of coded calibration scenes (CCS) to decrease the number of measurements in the calibration process [12]. Rather than scanning a single MNP sample at different positions, we use a number of coded scenes, each of which include multiple MNP samples at random positions. In the calibration procedure, the scenes are imaged, and then the SFM is reconstructed using these measurements with an augmented Lagrangian method [13]. Here, we analyse the effect of the number of scenes on the quality of the reconstructed images using simulations. We also compare the results with the standard single MNP sparse scanning method. 


\section{典刪曲艑}

Fig. 1. Numerical phantom (left), Reconstruction result with fully sampled SFM (right)

\section{METHODS}

\subsection{MPI System Parameters}

In order to generate the MPI signal, a two-dimensional (2D) forward model was simulated. The signal received from the MNPs was calculated using the equation below:

$$
u(t)=\int_{F O V}-\mu_{0} p^{R}(r) \cdot \frac{\partial \bar{m}(r, t)}{\partial t} c(r) d^{3} r
$$

where $c$ is the MNP distribution in the FOV, $p^{R}$ is the sensitivity of the receiver coils, $\mu_{0}$ is the free space magnetic permeability, $\bar{m}$ is the average of the magnetic moment of MNPs [14].

Magnetic field gradients were set as $3.75 \mathrm{~T} / \mathrm{m}$ and 1.875 $\mathrm{T} / \mathrm{m}$ in y and $\mathrm{z}$ directions, respectively. A $20 \mathrm{~mm} \times 10 \mathrm{~mm}$ FOV was used with $80 \times 40$ pixels. MNPs were assumed to be monodisperse with $30 \mathrm{~nm}$ diameter. Amplitude and base frequency $\left(f_{b}\right)$ of the drive field were set as $18 \mathrm{mT}$ and 2.5 $\mathrm{MHz}$, respectively. The field free point (FFP) moves on a Lissajous trajectory with $f_{b} / 96$ in the y direction and with $f_{b} / 99$ in the $\mathrm{z}$ direction [10]. The sampling frequency was 20 $\mathrm{MS} / \mathrm{s}$ and the signal was filtered with an ideal bandpass filter which passes $30 \mathrm{kHz}-1 \mathrm{MHz}$ band. The signal-to-noise ratio (SNR) of the signal was set to $20 \mathrm{~dB}$ using the awgn function of MATLAB.

The numerical phantom used in the simulations, and the reconstruction result with a fully sampled system calibration are presented in Fig. 1.

\subsection{Standard Compressed Sensing of the System Func- tion Matrix}

In the standard method for sparse reconstruction of the SFM [10], a single point source is moved to random pixels for the calibration measurements. The SFM is reconstructed with iterative convex optimization methods using the information that the system functions are sparse in the discrete cosine transform (DCT) domain [9]. In this work, Alternating Direction Method of Multipliers (ADMM) was used as a convex optimization method due to its fast convergence rate and accuracy [13]. In order to reconstruct the SFM, the following problem is solved:

$$
\begin{array}{ll}
\underset{\mathbf{A}}{\operatorname{argmin}} & \left\|\mathbf{D A}^{T}\right\|_{1} \\
\text { subject to } & \left\|\mathbf{A M}-\mathbf{A}_{\mathbf{s}}\right\|_{2}<\epsilon_{s},
\end{array}
$$

where $\mathbf{A}$ is the SFM reconstructed with ADMM in DCT domain, $\mathbf{D}$ is the DCT matrix, $\mathbf{M}$ is the undersampling matrix or mask, and $\mathbf{A}_{\mathbf{s}}$ is the SFM obtained by measuring the single MNP sample at different random positions on the grid.

In this work, we define the term "acceleration" as the total number of pixels $(\mathrm{N})$ divided by the number of measurements (e.g., five times acceleration means taking N/5 measurements for calibration). For the standard method, we used different masks corresponding to $1.25,2$, and 5 times acceleration rates as shown in Fig. 2a, 2b, and 2c, respectively.

For all the equations as above, $\|\cdot\|_{2}$ represents Frobenius norm of a matrix, $\|\cdot\|_{1}$ represents sum of absolute value of matrix elements, and $[\cdot]^{T}$ represents transform of a matrix.

\subsection{Proposed Method for Fast System Calibration}

The proposed method suggests the measurement of multiple coded scenes for calibration. The positions of the source points (i.e., the coding) are different for each scene, while the number of the source points are the same. This corresponds to sensing a linear combination of the columns of the original SFM. Using these measurements, SFM can be reconstructed using ADMM.

In order to reconstruct the SFM, the problem below is addressed:

$$
\begin{array}{ll}
\underset{\mathbf{A}}{\operatorname{argmin}} & \left\|\mathbf{D A}^{T}\right\|_{1} \\
\text { subject to } & \left\|\mathbf{A P}-\mathbf{A}_{\mathbf{p}}\right\|_{2}<\epsilon_{p},
\end{array}
$$

where $\mathbf{P}$ is the binary scene coding, and $\mathbf{A}_{\mathbf{p}}$ is the SFM sensed with $\mathbf{P}$.

In this study, $40 \%$ of the pixels were filled with MNP samples at each scene. The rows of the $\mathbf{P}$ matrix represent these CCSs. An example of a CCS is given in Fig. 2d. The number of measured scenes used in this study corresponds to 10, 20, and 33 times acceleration rates.

Compared to single MNP sample calibration, the use of multiple samples in the scenes improves the conditioning of the compressed sensing problem and increases the signal strength superlinearly with addition of each MNP sample. Eventually, the SNR is much higher during the calibration process because of adding multiple sources. However, the probability of having a noncoherent information after some filling rate will decrease [12].

\subsection{Image Reconstruction}

For image reconstruction using ADMM, a weighted sum of the total variation (TV) and the $l_{1}$ norm of the reconstructed image was minimized. This is suitable for MPI images, as 


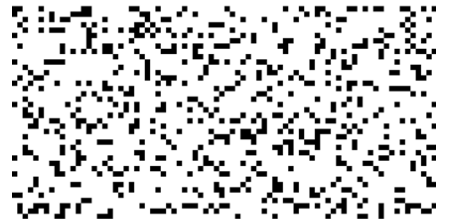

(a)

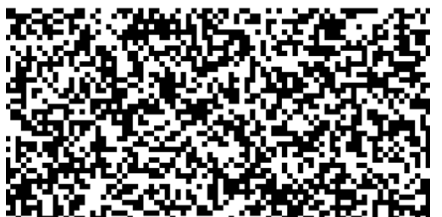

(b)

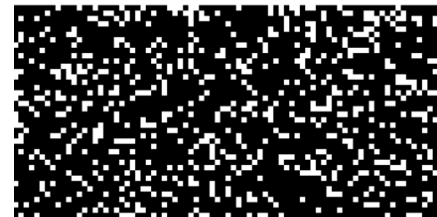

(c)

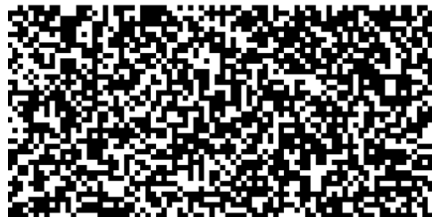

(d)

Fig. 2. Sampling masks for the case of using a single MNP sample for (a) 1.25, (b) 2, and (c) 5 times accelerations. The MNP sample is placed at one of the white pixel locations and scanned, and then moved to another white pixel location. (d) One of the coded scenes having $40 \%$ of the grid samples placed. Here, MNP samples are placed at all white pixel locations, at the same time. A different coded scene is used in the next step of calibration.

Standard Method
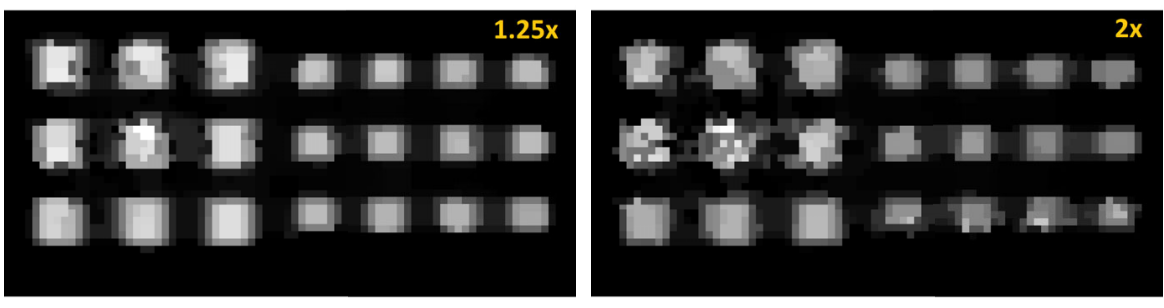

Proposed Method
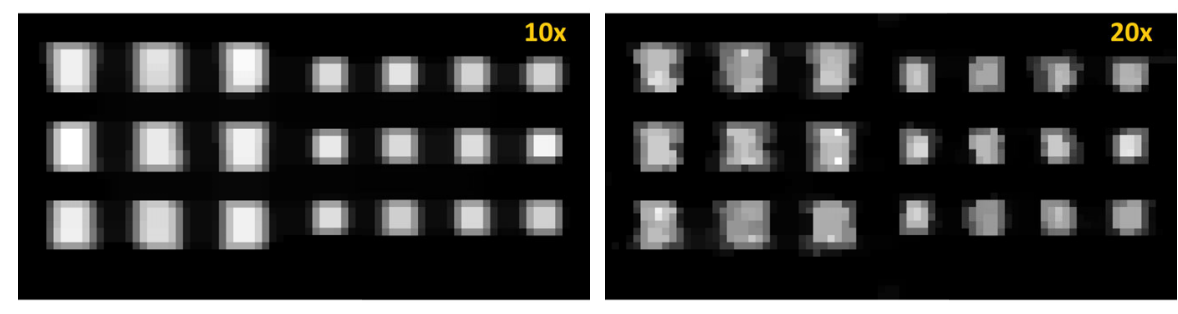
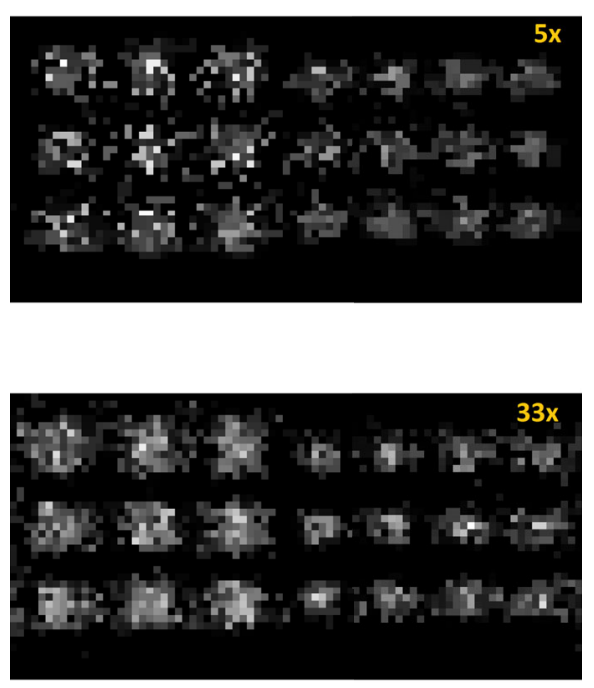

Fig. 3. Image reconstruction results with different reconstructed SFMs accelerated 1.25, 2, and 5 times using the standard method, and with system matrices accelerated 10, 20, and 33 times using the proposed method. The proposed method of using coded scenes enables significantly higher accelerations rates.

they are typically sparse by nature [15] and they have blockwise contiguous features that motivate the use of total variation. In addition, a nonnegativity constraint is added to the problem definition as negative pixels are not expected in MPI images. The convex optimization problem was formulated as follows [13]:

$$
\begin{array}{ll}
\underset{\mathbf{c}}{\operatorname{argmin}} & \alpha_{l_{1}}\|\mathbf{c}\|_{1}+\alpha_{T V} \mathrm{TV}(\mathbf{c}) \\
\text { subject to } & \|\mathbf{A} c-b\|_{2}<\epsilon, \\
& c_{i} \geq 0, i=\{1, \ldots, N\}
\end{array}
$$

where $b$ is the Fourier transform of the received signal, $\mathbf{c}$ is the desired 2D image, $c$ is the vectorized representation of c, $N$ is the number of pixels in the image $(N=3200$ for the examples in this work), and $c_{i}$ is the $i^{t h}$ pixel of $c$. The weighting parameters $\alpha_{l_{1}}$ and $\alpha_{T V}$ are adjusted to attain the best images that are evaluated by visual inspection.

\section{RESULTS}

Images obtained with the SFMs reconstructed with different methods and acceleration rates are shown in Fig. 3. The acceleration rates are emphasized on the top-right corners of the reconstructed images.

With 10 times acceleration in system calibration using the proposed CCS method, we obtained a better result compared to 1.25 times acceleration using the standard method. A similar comment can be made by comparing the results of 20 and 33 times acceleration using the CCS method with the results of 2 and 5 times acceleration using the standard method.

Structural similarity index (SSIM) and Peak SNR (PSNR) of each image were calculated (Table 1). The ideal numeric phantom shown in Fig. 1 is used as the reference image. 


\begin{tabular}{|c|c|c|c|c|c|c|}
\hline & \multicolumn{3}{|c|}{$\begin{array}{c}\text { Standard } \\
\text { Method }\end{array}$} & \multicolumn{3}{c|}{$\begin{array}{c}\text { Coded Scenes } \\
\text { Method }\end{array}$} \\
\hline Acc. & 1.25 & 2 & 5 & 10 & 20 & 33 \\
\hline SSIM & 0.71 & 0.56 & 0.31 & 0.79 & 0.60 & 0.36 \\
\hline PSNR & 13.0 & 10.4 & 7.71 & 14.48 & 11.78 & 8.87 \\
\hline
\end{tabular}

Table 1. SSIM and PSNR of the reconstructed images for different system calibration acceleration rates.

\section{DISCUSSION}

In this work, we proposed a new method for fast system calibration in MPI. The method uses multiple MNP samples for each calibration measurement, which we call the coded calibration scenes (CCS). The results show that reconstructing the system function matrix with these measurements improves the quality of the reconstructed images significantly, and enable much higher rate of acceleration when compared to the standard sparse reconstruction methods.

Following a preliminary analysis, a $40 \%$ filling rate was used in each CCS. The optimum filling rate for each CCS is the subject of future studies.

When comparing the acceleration rates between the standard and the CCS methods, we omitted the time delay originating from the mechanical movements between the measurements. The amount of time for a sample to move to the next sampling grid for the standard method may not be equal to the time for placing the next CCS for the proposed case. However, a hardware design optimized for this new calibration method may presumably minimize this transition time.

\section{REFERENCES}

[1] J. Haegele, J. Rahmer, B. Gleich, J. Borgert, H. Wojtczyk, N. Panagiotopoulos, T. M. Buzug, J. Barkhausen, and F. M. Vogt, "Magnetic Particle Imaging: Visualization of Instruments for Cardiovascular Intervention," Radiology, vol. 265, no. 3, pp. 933-938, 2012.

[2] B. Zheng, M. P. von See, E. Yu, B. Gunel, K. Lu, T. Vazin, D. V. Schaffer, P. W. Goodwill, and S. M. Conolly, "Quantitative Magnetic Particle Imaging Monitors the Transplantation, Biodistribution, and Clearance of Stem Cells In Vivo," Theranostics, vol. 6, no. 3, pp. 291-301, 2016.

[3] J. Yang, J. Gunn, S. R. Dave, M. Zhang, Y. A. Wang, and X. Gao, "Ultrasensitive detection and molecular imaging with magnetic nanoparticles," Analyst, vol. 133, no. 2, pp. 154-160, 2008.

[4] J. R. McCarthy and R. Weissleder, "Multifunctional magnetic nanoparticles for targeted imaging and ther- apy," Adv Drug Deliv Rev., vol. 60, no. 11, pp. 12411251, 2008.

[5] B. Gleich and J. Weizenecker, "Tomographic imaging using the nonlinear response of magnetic particles," $\mathrm{Na}$ ture, vol. 435, no. 7046, pp. 1214-1217, 2005.

[6] P. W. Goodwill, E. U. Saritas, L. R. Croft, T. N. Kim, K. M. Krishnan, D. V. Schaffer, and S. M. Conolly, "XSpace MPI: Magnetic Nanoparticles for Safe Medical Imaging," Adv. Mater., vol. 24, pp. 3870-3877, 2012.

[7] T. Knopp, J. Rahmer, T. F. Sattel, S. Biederer, J. Weizenecker, B. Gleich, J. Borgert, and T. M. Buzug, "Weighted iterative reconstruction for magnetic particle imaging," Phys. Med. Biol., vol. 55, no. 6, pp. 15771589, 2010.

[8] J. Weizenecker, B. Gleich, J. Rahmer, H. Dahnke, and J. Borgert, "Three-dimensional real-time in vivo magnetic particle imaging," Phys. Med. Biol., vol. 54, no. 5, pp. L1-L10, 2009.

[9] J. Lampe, C. Bassoy, J. Rahmer, J. Weizenecker, H. Voss, B. Gleich, and J. Borgert, "Fast reconstruction in magnetic particle imaging," Phys Med Biol, vol. 57, no. 4, pp. 1113-1134, 2012.

[10] T. Knopp and A. Weber, "Sparse Reconstruction of the Magnetic Particle Imaging System Matrix," IEEE Trans. Med. Imag., vol. 32, no. 8, pp. 1473-1480, 2013.

[11] A. Weber and T. Knopp, "Reconstruction of the Magnetic Particle Imaging System Matrix Using Symmetries and Compressed Sensing," Adv. Math. Phys., vol. 2015, pp. 460496, 2015.

[12] G. R. Arce, D. J. Brady, L. Carin, H. Arguello, and D. S. Kittle, "Compressive Coded Aperture Spectral Imaging," IEEE Signal Processing Magazine, vol. 31, no. 1, pp. 105-115, 2014.

[13] S. Ilbey, C. B. Top, T. Çukur, E. U. Saritas, and H. E. Güven, "Image reconstruction for magnetic partical imaging using an augmented lagrangian method," in International Symposium on Biomedical Imaging, 2017.

[14] T. Knopp and T. M. Buzug, Magnetic Particle Imaging: An Introduction to Imaging Principles and Scanner Instrumentation, Springer, Berlin/Heidelberg, 2012.

[15] P. W. Goodwill and S. M. Conolly, "Multidimensional X-Space Magnetic Particle Imaging," IEEE Trans. Med. Imag., vol. 30, no. 9, pp. 1581-1590, 2011. 